\title{
Anschriften der Autoren
}

Prof. Dr. Werner Abraham

Maitschern 128

A-8942 Wörschach

Dr. Hardarik Blühdorn

Institut für Deutsche Sprache

Postfach 101621

D-68016 Mannheim

Prof. Dr. Andreu Castell

Universitat Rovira i Virgili

Facultat de Lletres

Dpt. De Filologia Anglogermànica

Plaça Imperial Tarraco, 1

E-43005 Tarragona

Prof. Dr. Martine Dalmas

Université Paris IV-Sorbonne

Centre Universitaire Malesherbes

108, boulevard Malesherbes

F-75850 Paris Cedex 17

Prof. Dr. Ludwig M. Eichinger

Institut für Deutsche Sprache

Postfach 101621

D-68016 Mannheim

Prof. Dr. Martin Durell

The University of Manchester

Department of German

Oxford Road

Manchester M13 9PL

Großbritannien 
Prof. Dr. Cathrine Fabricius-Hansen

Universität Oslo

Germanistisches Institut

Postfach 1004

$\mathrm{N}-0315$ Oslo 3-Blindern

Prof. Dr. Csaba Földes

Universität Veszprem

Lehrstuhl für deutsche Sprache und Literatur

Füredi u. 2

H-8201 Veszprem

Prof. Dr. Regina Hessky

Eötvös Lorand Universität

Philologische Fakultät

Germanistisches Institut

Ajtosi Dürer sor 19/21

H-1 146 Budapest

Prof. Dr. Irma Hyvärinen

Universität Helsinki

Germanistisches Institut

Unioninkatu 40, Zi. C521

FIN-00014 Helsinki

Prof. Dr. Hans-Jürgen Krumm

Universität Wien

Institut für Germanistik

Deutsch als Fremdsprache

Mariahilferstraße 88a/1/6

A-1070 Wien

Prof. Dr. Claudio Di Meola

Università degli Studi di Roma

„La Sapienza“

Facoltà di Lettere e Filosofia

Via Carlo Fea 2

I-00161 Rom

Prof. Dr. Giuliana Liebman Parrinello

Dipartimento di Letterature Comparate

Università degli Studi Roma Tre

Via de Castro Pretorio, 20

I-00185 Roma 
Prof. Dr. Angelika Redder

Institut für Deutsch als Fremdsprache

der Universität München

Ludwigstraße 27/I

D-8000 München

Prof. Dr. Shrishail Sasalatti

Centre of German Studies

SLI\&CS, J. Nehru University

New-Delhi

IND-110067

Prof. Dr. Speranta Stanescu

Al. Parva 10, bl D 23, Sc. B, Ap. 14

RO-77429 Bukarest

Prof. Dr. Gerhard Stickel

Obere Bergstraße 32

D-69198 Schriesheim

Prof. Dr. Christiane von Stutterheim

Institut für Deutsch als Fremdsprachenphilologie

der Universität Heidelberg

Plöck 55

69117 Heidelberg

Prof. Dr. Michael Townson

60 Blenheim Road

Birmingham B 139TZ

Großbritannien

Prof. Dr. Joachim Umlauf

Deutscher Akademischer Austauschdienst

Grundsatzabteilung

Kennedyallee 50

D-53175 Bonn

Prof. Dr. Gisela Zifonun

Institut für Deutsche Sprache

Postfach 101621

D-68016 Mannheim 